\title{
Integrated Use of Time-Frequency Wavelet Decompositions for Fault Location in Distribution Networks: Theory and Experimental Validation
}

\author{
A. Borghetti, Senior Member, IEEE, M. Bosetti, C. A. Nucci, Fellow, IEEE, M. Paolone, Member, IEEE, and
} A. Abur, Fellow, IEEE

\begin{abstract}
The paper presents a procedure for fault location in distribution networks, based on the use of the integrated time-frequency wavelet decompositions of the voltage transients associated with the fault-originated travelling waves. The proposed analysis of time-frequency wavelet decompositions has been found to improve the identification accuracy of the frequencies associated to the characteristic patterns of a fault location with respect to a sole frequency-domain wavelet analysis. Several laboratory fault tests, carried out by means of a reduced-scale model of a distribution feeder, are used to illustrate the characteristics and assess the performances of the proposed improved procedure. The paper also illustrates the application of the proposed procedure to a transient, originated by a permanent phase-to-phase fault, measured in a real distribution network in which a post-test analysis has identified the faulted branch.
\end{abstract}

Index Terms-Continuous wavelet transform, distribution networks, fault location, fault transients, power quality.

\section{INTRODUCTION}

$\mathbf{T}$ HE accurate fault location in medium-voltage (MV) distribution networks is one of the aspects that has a major impact on the service continuity. Moreover, switching transients associated to both fault location searching techniques and subsequent service restoration reconfiguration maneuvers may also affect power quality of the distribution networks [1].

Various procedures for fault location assessment are described in the literature (e.g., [2], [3]). Some procedures analyze the fundamental waveforms of pre and post-fault voltages and currents (e.g., [4]-[6]), whilst others are based on the analysis of the fault-originated electromagnetic (EM) transients, i.e., the traveling waves generated by the fault itself (e.g., [7]-[16]).

The procedure presented in this paper belongs to the second of the above mentioned categories. In particular, it is aimed at determining the fault location by using solely the measured voltage transients in correspondence of the MV bus of the feeding substation of a radial distribution network without the need of multirecording points and relevant synchronization. Indeed, the high-

Manuscript received December 21, 2009; revised February 17, 2010. First published April 29, 2010; current version published September 22, 2010. Paper no. TPWRD-00952-2009.

A. Borghetti, M. Bosetti, C.A. Nucci, and M. Paolone are with the Department of Electrical Engineering, University of Bologna, Bologna, Italy (e-mail: alberto.borghetti; carloalberto.nucci; mauro.bosetti; mario.paolone@unibo.it).

A. Abur is with the Electrical and Computer Engineering Department, Northeastern University, Boston, MA 02115 USA (e-mail: abur@ece.neu.edu).

Digital Object Identifier 10.1109/TPWRD.2010.2046655 frequency transient recorded at a certain bus of a faulted feeder, and triggered by the fault itself, carries useful information associated to the relevant fault location. These information refers to the sequence of reflection/transmission phenomena, taking place in correspondence of the network discontinuities and terminations, which affect the fault-originated EM travelling waves. In particular, as illustrated in [13]-[16], the paths followed by the fault-originated EM travelling waves can be associated to characteristic frequencies identified by means of their energy content estimated using the wavelet analysis. ${ }^{1}$

In [13] and [15], use is made of wavelet packets to process the high-frequency wave signals, whilst as in [14] and [16], the procedure proposed in this paper is based on the continuous wavelet analysis (CWT) of fault-originated transients by using fault-originated mother wavelets [16].

As is known (e.g., [17]), the numerical implementation of the CWT applied to a sampled signal $s(t)$, which in our case corresponds to a first portion of the recorded voltage fault transient, provides a matrix $C$ whose elements are

$$
\begin{aligned}
C(a, b) & =C\left(a, i T_{s}\right) \\
& =T_{s} \frac{1}{\sqrt{|a|}} \sum_{n=0}^{N-1}\left[\psi^{*}\left(\frac{n T_{s}-i T_{s}}{a}\right) s\left(n T_{s}\right)\right]
\end{aligned}
$$

where:

$-T_{s}$ is the sampling time and $N$ is the number of recorded samples of $s(t)$;

- $a$ corresponds to the so-called scale factor;

$-b=i \cdot T_{s}$ corresponds to the so-called time shifting factor, being $i$ an integer value;

$-\psi(a, b)^{*}$ is the complex conjugate of the so-called daughter wavelet $\psi$, which is a time translated and scale expanded/ compressed version of a finite energy function $\Psi(t)$, called a mother wavelet.

As largely discussed in the literature, coefficients $C(a, b)$ can be seen as "similarity indices" between the signal and daughter wavelets $\psi$ located at time position $b$ with scale $a$. If the center frequency of the mother wavelet $\psi(t)$ is $F_{0}$, the one of the

${ }^{1}$ It is worth reminding that transient signals associated to faults are characterized by time-variant characteristics that produce a continuous spectrum of the signal in the frequency domain. Therefore, the identification of the characteristic frequencies by means of traditional operators, such as the fast Fourier transform (FFT), may be considered in general as inadequate. The identification of the characteristic frequencies should be therefore accomplished by using appropriate signal analysis techniques that allow the adjustment of the signal spectrum versus time. In this respect, the wavelet analysis is one of these techniques. 
daughter-wavelet $\psi(a t)$ is $F_{0} / a$. Therefore, coefficients $C(a, b)$ represent a bidimensional function of time and frequency.

In [14] and [16], only the frequency-domain information is used for the fault location, i.e., the characteristic frequency associated with the fault location are identified by inspecting the local maxima of the CWT signal energy $E_{\mathrm{CWT}}$ calculated as the sum of the squared values of all coefficients belonging to the same scale $a$

$$
E_{\text {cwt }}(a)=\sum_{n=0}^{N-1}\left(C\left(a, n T_{s}\right)\right)^{2} .
$$

As discussed in [18], $E_{\mathrm{CWT}}$ identifies a "scalogram", which provides the weight of each frequency component.

However, the use of classical mother wavelets (e.g., the Morlet one) does not allow, in general, the identification of all the frequencies associated to the fault-originated travelling-wave paths. As shown in [16], this limitation can be overcome by means of an appropriate definition of the mother wavelet which, to maximize the similarity indexes between the analyzed signal and the daughter-wavelet, can be inferred from fault transient itself.

In order to significantly improve the algorithm performances, i.e., to obtain a more precise identification of the characteristic frequencies and therefore reduce the accuracy of the fault location identification, this paper proposes to integrate the frequency-domain information with the time-domain information provided by the CWT analysis. In particular, the paper proposes a two step approach: 1) identification of the frequencies $f_{i}$ associated with the local maxima of the wavelet transform energy scalogram as done in [16] and 2) improved identification of $f_{i}$ by inspecting time differences between consecutive maxima of the wavelet transform coefficients $C(a, b)$ within a frequency interval $f_{i} \pm \Delta f$ (e.g., [10] and [11]).

The structure of the paper is the following. As the proposed CWT analysis takes into account the peculiar characteristics of voltage transients measured at a bus of a faulted feeder, Section II first anticipate the description of the reduced-scale experimental setup that makes reference to typical cable-type distribution network configurations. Section III presents the proposed procedure and Section IV is devoted to the validation of the procedure with reference to several faults events emulated by using the reduced-scale experimental setup and also for the case a permanent fault event occurred, and measured, in a real distribution network. Section V concludes the paper.

\section{REDUCED-SCALE EXPERIMENTAL SETUP}

Fault location algorithms based on the analysis of the faultoriginated EM transients are often tested by using the results of numerical simulations carried out by means of electromagnetic transient programs (EMTP) [19] able to accurately represent the propagation phenomena that takes place in line feeders (e.g., [20], [21]).

However, in order to extend the application of the proposed fault location algorithm also to real electromagnetic fault transients, in this paper they are experimentally obtained by means

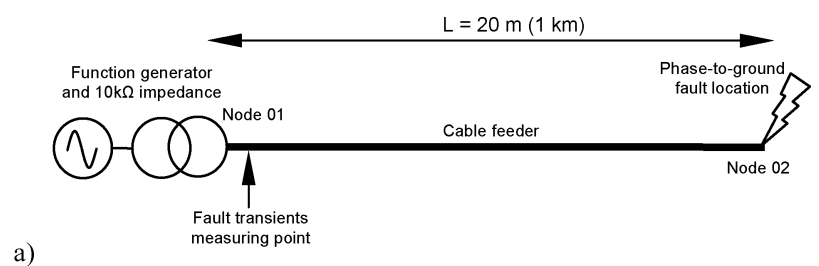

a)

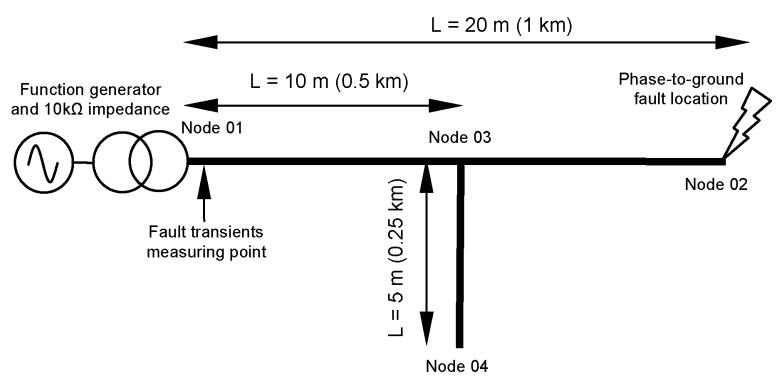

Fig. 1. Topologies of the reduced-scale setup aimed at reproducing electromagnetic fault transients: (a) single feeder and (b) feeder including a lateral branch. Real scale lengths are reported in parenthesis.

of a reduced-scale experimental setup aimed at reproducing the fault-transient response of single-phase cable feeders.

Two topologies have been taken into consideration, both illustrated in Fig. 1: a) a single 1-km-long line and b) a 1-km-long main feeder with a 250-m-long lateral branch connected at the middle of the main (real scale length).

The assumed scale factor is of 1:50. All cable lengths are divided by this scale factor whilst the frequency of the power supply is multiplied by the scale factor in order to keep constant the ratio between the feeding voltage wavelength and the cable lengths. The reduced-scale cable lengths are reported in Fig. 1 as well as, in parenthesis, the real-scale ones. The equivalent reduced-scale power supply frequency, for a real-scale rated frequency of $50 \mathrm{~Hz}$, is of $2.5 \mathrm{kHz}$.

Each line is emulated by a single standard RG58 shielded cable, characterized by a $50-\Omega$ surge impedance and a measured propagation speed of $1.78610^{8} \mathrm{~m} / \mathrm{s}$.

The feeding voltage is provided by an Agilent 33120 A function generator placed in series with a lumped $10-\mathrm{k} \Omega$ resistance that represents, as a first approximation, the primary substation transformer response to incoming fault-generated travelling waves. ${ }^{2}$

The fault between the cable shield and its inner conductor is generated by means of a fast TTL-controlled micro-switch triggered by a National Instruments 9401 high-speed digital I/O board.

The fault-originated waveforms are recorded in a single point located in correspondence of the junction between the cable feeder and the 10-k $\Omega$ lumped impedance, this measurement point represents, in the real scale, the medium voltage side of the primary substation transformer. The signals are recorded by means of a LeCroy LT264 8-bit digital oscilloscope operating at the sampling frequency of $1 \mathrm{GHz}$.

\footnotetext{
${ }^{2}$ The response of power distribution transformers against incoming high-frequency travelling waves is typically similar to that of an open-end termination. Therefore, in the reduced-scale setup, each transformer is represented by means of a 10-k $\Omega$ lumped resistance, much larger than the cable surge impedance (50 $\Omega$ ).
} 


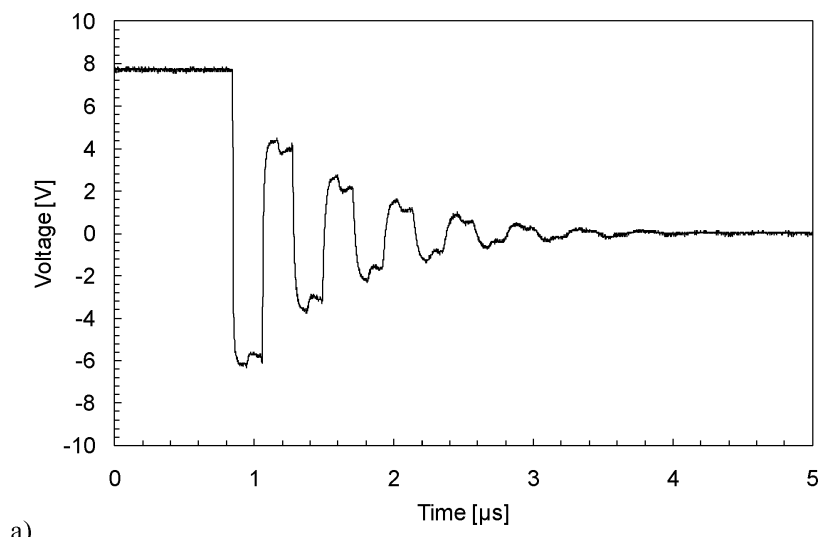

a)

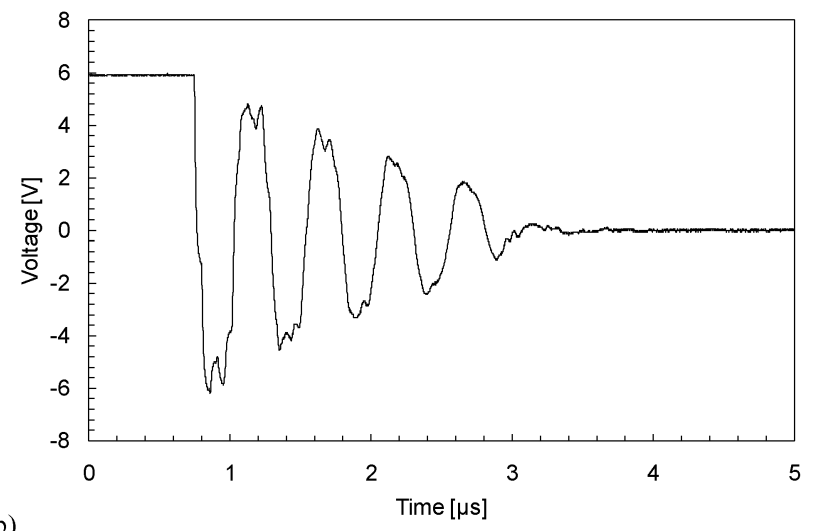

b)

Fig. 2. Reduced scale voltage transients measured in correspondence of the node 01 of Fig. 1: (a) configuration of Fig. 1(a) and (b) configuration of Fig. 1(b).

\section{INTEGRATED USE OF TIME-FREQUENCY WAVELET DECOMPOSITION APPLIED TO FAULT TRANSIENTS}

As in [14] and [16], the fault location procedure is based on the identification of the characteristic frequencies associated with the specific paths followed by the fault-originated travelling waves to the to the measurement point.

Assuming known both the network topology and the traveling wave speed $v_{i}$ of each propagation mode $I$ [19], [22], frequency $f_{p, i}$ of mode $i$ through path $p$ is

$$
f_{p, i}=\frac{v_{i}}{n_{p} L_{p}}
$$

where $L_{p}$ is the length of path $p$ and $n_{p}(\in \mathbf{N})$ is the number of times a given travelling wave propagates along path $p$ before attaining the same polarity.

The application of (3) allows to recognize $p-1$ values used to identify the faulted section and the remaining one used to identify the distance between observation point $m$ and the fault location [14], [16].

Coefficient $n_{p}$ depends on the sign of the reflection coefficients associated to the terminals of the two ends of the specific travelled path $p$. This implies that $n_{p}$ is equal to 2 or 4 if the sign of the reflection coefficients are the same or different respectively. In particular, terminals where a power transformer is connected can be considered, for the traveling waves, as open circuits with reflection coefficient close to +1 , whereas terminals that appear as junctions between more than two lines will have a negative reflection coefficient. Furthermore, the fault point can be assumed to have a reflection coefficient close to -1 .
TABLE I

Characteristic Frequencies ReleVANT to the Propagation Paths oF TOPOLOGIES RePorted IN Fig. 1 (REDUCED SCALE CABLE LENGTH)

\begin{tabular}{|c|c|c|}
\hline \multicolumn{3}{|c|}{ "Single feeder (configuration shown by Fig. 1a) } \\
\hline Path & $\begin{array}{c}\text { Path length } \\
n_{p} \cdot L_{p} \\
{[\mathrm{~m}]}\end{array}$ & $\begin{array}{c}\text { Theoretical frequencies } f_{p, i} \\
\text { (inner-to-shield cable traveling speed equal } \\
\text { to } 1.786 \cdot 10^{8} \mathrm{~m} / \mathrm{s} \text { ) } \\
{[\mathrm{MHz}]}\end{array}$ \\
\hline Node 01-02 & $4 \times 20\left(n_{p}=4\right)$ & 2.233 \\
\hline \multicolumn{3}{|c|}{ Feeder including a lateral branch (configuration shown by Fig. 1b) } \\
\hline Node 01-02 & $4 \times 20\left(n_{p}=4\right)$ & 2.233 \\
\hline Node $01-03$ & $4 \times 10\left(n_{p}=4\right)$ & 4.465 \\
\hline Node $01-04$ & $2 \times 15\left(n_{p}=2\right)$ & 6.953 \\
\hline
\end{tabular}

Fig. 2 shows the fault-originated voltage transients, measured at the node 01, corresponding to the two topologies of Fig. 1. It can be observed that, as the fault triggered by the TTL microswitch can be assumed as a step-function source, the voltage transients of Fig. 2 can be seen as the result of the superposition of different square waves, each one having a fundamental frequency given by (3), in addition to the steady state voltage waveform. This behavior is particularly clear for the case of Fig. 2(a) which corresponds to the single cable feeder.

Table I shows the theoretical frequencies observed at node 01 of Fig. 1 obtained by applying (3) to the two considered network configurations.

As mentioned in the Introduction, the identification of the characteristic frequencies is not straightforward as the fault transient is characterized by a continuous spectrum due to its timevariant properties. The use of the CWT allows an improved identification of these frequencies as it provides a good frequency resolution at low frequencies and a good time resolution at high frequencies [17], characteristics that allow the identification of high-frequency components very close in time and low frequency components very close in frequency. ${ }^{3}$

In order to overcome some limitations relevant to the use of traditional mother wavelets (e.g., the Morlet one), in this paper the CWT analysis has been performed by using fault-inferred mother wavelets using the approach proposed in [16].

As mentioned in the Introduction, in [14] and [16] the identification of the characteristic frequencies $f_{p, i}$ associated to the fault location was realized by inspecting the relative maximum peaks of the obtained scalogram $E_{\text {cwt }}(a)$ provided by (2). Such an approach disregards the information provided by the CWT time decomposition that can also be used to successfully locate the fault as proposed in [10] and [11].

The improvement introduced by the method here proposed is due to the integrated time-frequency analysis which involves a two-step identification of the characteristic frequencies.

The first step consists of an initial estimation of such frequencies $f_{p, i}^{I}$ as was done in [14] and [16].

\footnotetext{
${ }^{3}$ The presence of harmonic distortion in the voltage waveform has a minor influence on the frequencies $f_{p, i}$, as amplitudes of harmonic components of a distorted voltage waveform are typically limited to few percent of the fundamental frequency component, whilst fault transients are characterized by a continuous spectrum with component amplitudes comparable with the one of the fundamental component. Indeed, the limits set by the IEC 61000-3-6 standard [23] are $1.7 \%$ for odd non-multiple-of- 3 components, $0.2 \%$ for odd multiple-of- 3 components and below $0.36 \%$ for even components, in the interval between the 17 th to the 49th harmonic.
} 


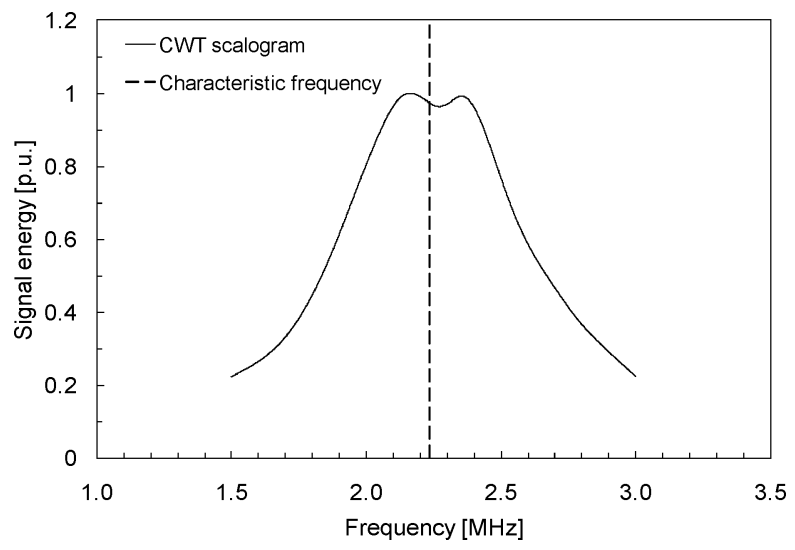

Fig. 3. Energy scalogram relevant to the CWT analysis of the fault transient of Fig. 2(a) relevant to the single cable configuration of Fig. 1(a).

The second step improves the initial estimate by identifying the time differences between local maxima of the signal coefficients $C(a, b)$ defined by (1) in a specific frequency range centered in correspondence of the frequency $f_{p, i}^{I}$ identified by the first step.

Let us consider the configuration shown in Fig. 1(a) (single cable feeder) as a first example to illustrate the use of the proposed procedure. As shown in Table I, this configuration has one characteristic frequency $f_{p, i}$ equal to $2.233 \mathrm{MHz}$. Fig. 3 shows the obtained scalogram relevant to the signal energy values $E_{\text {cwt }}(a)$ provided by (2) and by using the procedure described in [16]: a frequency $f_{p, i}^{I}$ of $2.162 \mathrm{MHz}$ is identified.

Denoting with $\tilde{p}$ the actual path between the observation point and the fault location, and with $L_{\tilde{p}}$ its length, in view of (3) the fault location error expression reads

$$
e_{\%}=\frac{100}{L_{\tilde{p}}}\left|L_{\tilde{p}}-\frac{\nu_{i}}{n_{\tilde{p}} \cdot f_{\tilde{p}, i}^{\mathrm{CWT}}}\right|
$$

where $f_{\tilde{p}, i}^{\mathrm{CWT}}$ is the CWT-identified frequency relevant to path $\tilde{p}$ and $n_{\tilde{p}}$ is equal to 4 for the this configuration. By means of (4) and the results of Table I, we have computed the percentage difference between the identified and the theoretical frequency values as well as the fault location error: they are equal to $3.2 \%$ and $3.3 \%$, respectively.

As illustrated above, the $f_{p, i}^{I}$ value of $2.162 \mathrm{MHz}$ is then used to explore time differences between local maxima in the signal coefficients $C(a, b)$. In particular, Fig. 4 shows $C(a, b)$ in the frequency range surrounding $f_{p, i}^{I}=2.162 \mathrm{MHz}$ with a $\Delta f=$ $0.25 \mathrm{MHz}$ that allows to identify a time difference of $0.4425 \mu \mathrm{s}$ that corresponds to an identified characteristic frequency of 2.26 $\mathrm{MHz}$. This yields a frequency error of $1.2 \%$ and a fault location error of $1.2 \%$. It is important to note that the identified time difference between local maxima of $C(a, b)$ remains constant in the considered frequency range.

As evident from the above results an important error reduction in the estimated the fault location can be achieved. In the following section, the application of the improved fault location procedure is extended to other topologies referring to both reduced scale configuration of Fig. 1(b) and for a real distribution network configuration.

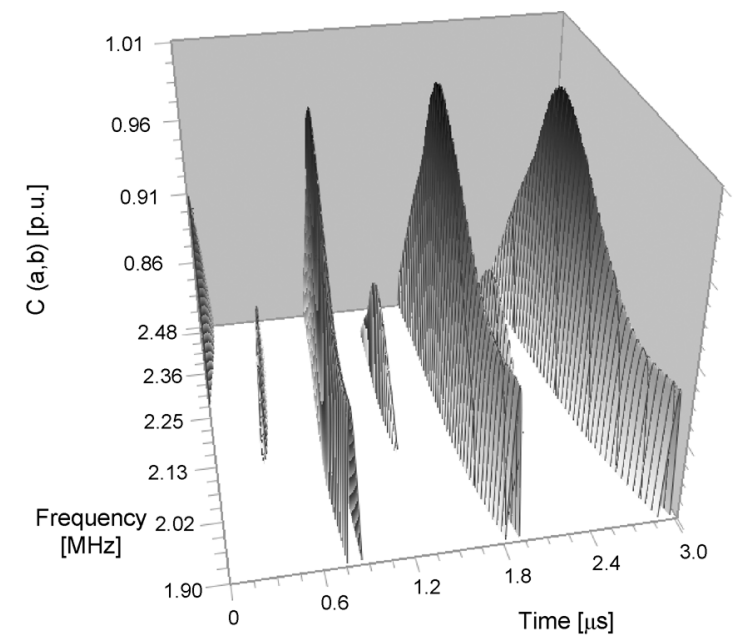

a)

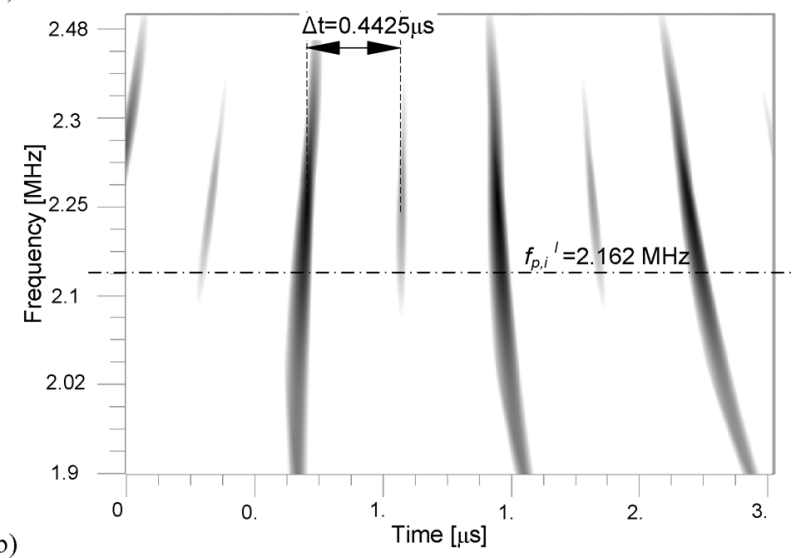

Fig. 4. Coefficients $C(a, b)$ obtained by means of the CWT analysis applied to the fault transient of Fig. 2(a): improved estimation of the estimation of the characteristic frequency associated to the faulted path between nodes $01-02$.

\section{EXPERIMENTAL VALIDATION}

This section of the paper contains further experimental validation of the proposed fault location procedure by making reference to more complex network topologies. In particular, two case studies are presented: the first one refers to the configuration of Fig. 1(b) and the second one to a fault transient recorded in a real distribution network.

\section{A. Reduced Scale Experimental Setup: Feeder Including a Lateral Branch}

Let consider the reduced scale network topology shown in Fig. 1(b) and the relevant fault transients of Fig. 2(b). Table I gives the three characteristic frequencies associated to the fault occurring at the end of the main feeder.

Fig. 5 shows the scalogram of the signal energy values $E_{\text {cwt }}(a)$ obtained using (2) to coefficients $C(a, b)$. The identified frequencies are shown in Table II. These results imply that the error in the identified characteristic frequency for the given fault location is equal to $11.3 \%$ and a fault location error equal to $12.7 \%$.

Similar to the case of the single cable feeder, Fig. 6 shows the analysis for the identification of time differences between local maxima of the coefficients $C(a, b)$ in the frequency range 


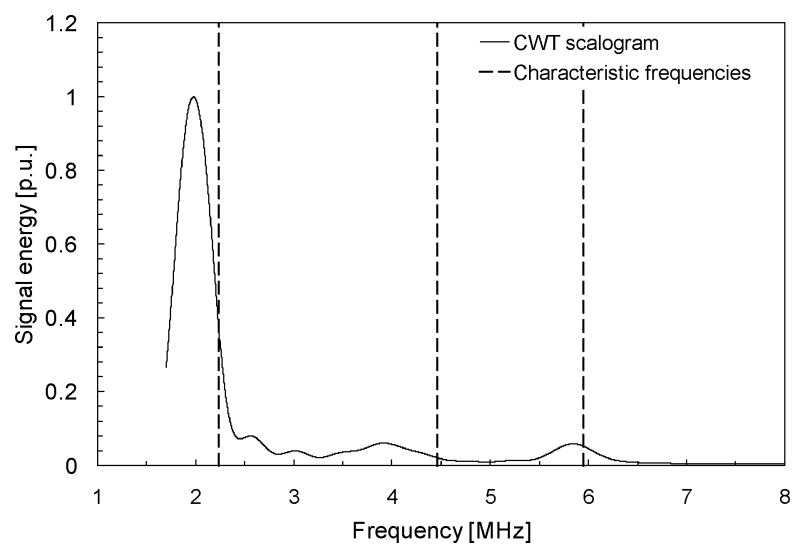

Fig. 5. Energy scalogram relevant to the CWT analysis of the fault transient of Fig. 2(b) relevant to the single cable configuration of Fig. 1(b).

TABLE II

CHARACTERISTIC AND CWT IDENTIFIED FREQUENCIES USING THE APPROACH OF [16] RELEVANT TO THE PROPAGATION PATHS OF TOPOLOGIES REPORTED IN Fig. 1(B) (REDUCED SCALE CABLE LENGTH)

\begin{tabular}{|c|c|c|c|}
\hline Path & $\begin{array}{c}\text { Path length } \\
n_{p} \cdot L_{p} \\
{[\mathrm{~m}]}\end{array}$ & $\begin{array}{c}\text { Theoretical } \\
\text { frequencies } f_{p, i} \\
\text { (traveling speed } \\
\text { equal to } \\
1.786 \cdot 10^{8} \mathrm{~m} / \mathrm{s} \text { ) } \\
{[\mathrm{MHz}]}\end{array}$ & $\begin{array}{l}\text { CWT identified } \\
\text { frequencies } f_{p, i} \\
\text { by using the } \\
E_{c w l}(a) \\
{[\mathrm{MHz}]}\end{array}$ \\
\hline Node 01-02 & $4 \times 20\left(n_{p}=4\right)$ & 2.233 & 1.980 \\
\hline Node 01-03 & $4 \times 10\left(n_{p}=4\right)$ & 4.465 & 3.922 \\
\hline Node 01-04 & $2 \times 15\left(n_{p}=2\right)$ & 5.953 & 5.844 \\
\hline
\end{tabular}

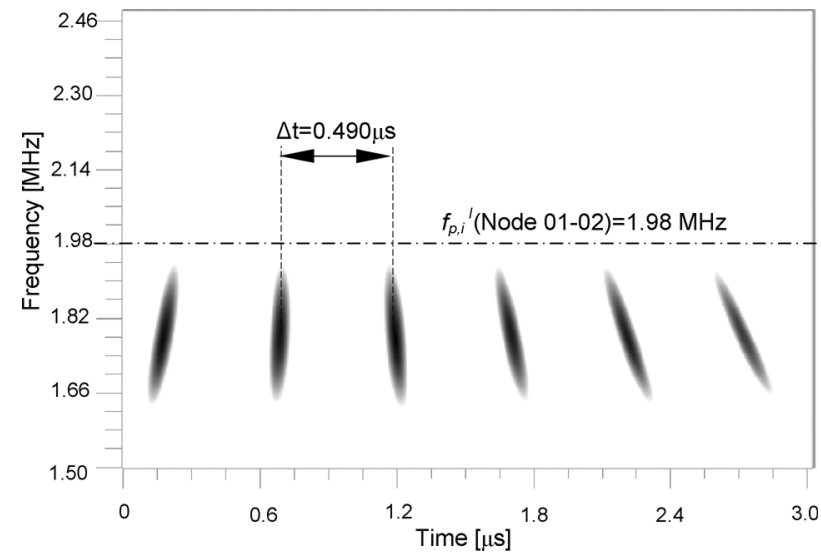

Fig. 6. Coefficients $C(a, b)$ obtained by means of the CWT analysis applied to the fault transient of Fig. 2(b): improved estimation of the characteristic frequency associated with the faulted path between nodes 01-02.

centered in the first-step identified frequency $f_{p, i}^{I}=1.980 \mathrm{MHz}$ that corresponds to the fault location path (node 01-node 02).

By making reference to this frequency, Fig. 6 shows coefficients $C(a, b)$ in the frequency range surrounding $f_{p, i}^{I}=1.980$ $\mathrm{MHz}$ with a $\Delta f=0.25 \mathrm{MHz}$ that allows to identify a time difference of $0.490 \mu \mathrm{s}$ corresponding to a characteristic frequency of $2.04 \mathrm{MHz}$. This yields a frequency error of $8.6 \%$ and a fault location error of $9.3 \%$. Hence, even in this more complex case, the proposed approach yields an improvement estimate for the location of the fault.

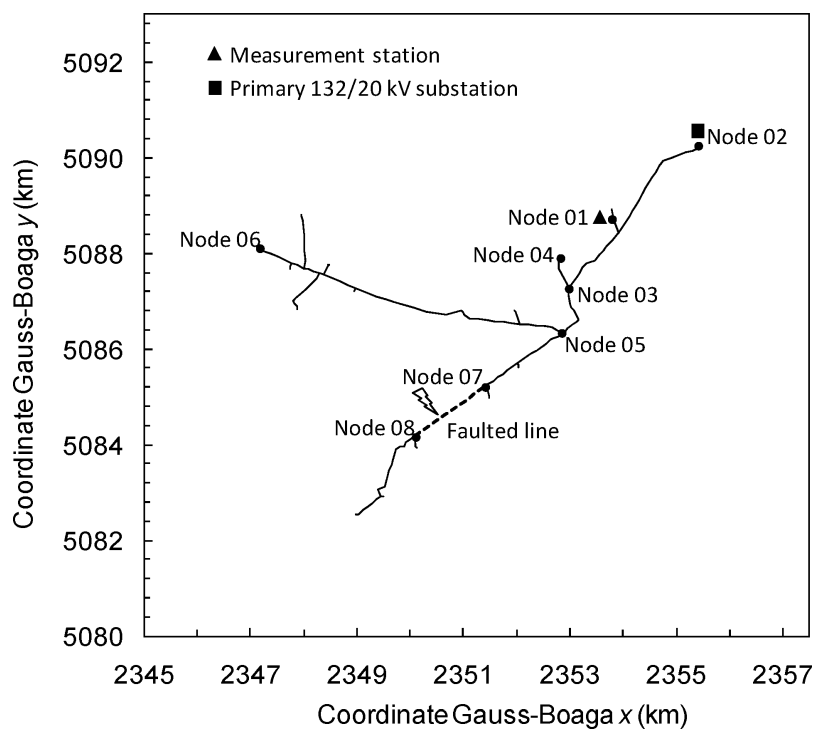

Fig. 7. Topology of the considered distribution network and point of interest.

\section{B. Fault Transients Recorded in a Real Distribution Network}

The proposed fault location procedure is also applied for locating a fault for which recorded electromagnetic transients were captured by means of the monitoring system described in [24], [25]. This monitoring system was suitably designed to measure lightning-originated overvoltages and it was installed at some secondary substations of a distribution network located in the northern region of Italy. It has the necessary sensor bandwidth $(4 \mathrm{MHz})$ and sampling frequency $(100 \mathrm{MSa} / \mathrm{s})$ in order to accurately measure the electromagnetic transients originated by fault events [24]. The measurement system also provides, for each event, the relevant time of occurrence recorded by triggering a Universal Time Code-Global Positioning System (UTC-GPS) unit characterized by an overall time uncertainty of $100 \mathrm{~ns}$.

The monitored distribution network line is one of the 13 feeders that starts from a common primary $132 / 20-\mathrm{kV}$ substation. The distribution network operates with isolated neutral grounding. The considered feeder is composed of three-phase overhead lines of overall length equal to $20.3 \mathrm{~km}$ and $1.3 \mathrm{~km}$ of overhead and buried shielded cables.

The overhead lines consist of three conductors (without a shield wire), located at $10 \mathrm{~m}, 10.8 \mathrm{~m}$ and $10 \mathrm{~m}$ above ground, respectively. The feeder is composed of three main branches, each 7-km long, arranged in a configuration shown in Fig. 7. The figure shows the network topology in plane coordinates using the Gauss-Boaga $x-y$ reference system.

The feeder is protected with one circuit breaker equipped with a three-level overcurrent relay (for multiphase faults) and a three-level zero-sequence relay (for line-to-ground faults).

In the considered feeder, an additional monitoring system for protection manoeuvres is also installed at the primary substation [26]. It consists of a PC-based system that records the status of the different feeder protection devices, namely: overcurrent, 0-sequence and circuit-breaker operation relays. The system records any change of status relevant to each protection device into an ASCII file that can be post-processed. In the 


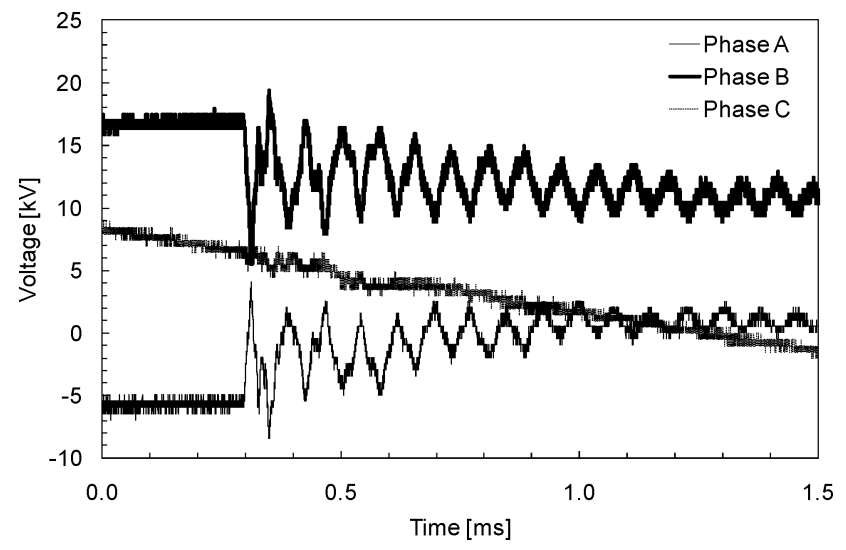

Fig. 8. Voltage transient observed in correspondence of node 01 due to a fault between nodes $07-08$ of Fig. 7.

same record, the system also provide the relevant time of occurrence using the relevant UTC value by means of a GPS unit characterized by a time uncertainty of $10 \mathrm{~ms}$. The availability of both UTC-GPS times of both measured transients and protection status changes, allows to correlate each protection manoeuvre intervention to a specific recorded transient.

The system is in operation since 2007 and several fault events have been recorded. One of them, recorded on July 24, 2007 at $18 \mathrm{~h} 25 \mathrm{~m} 14 \mathrm{~s}$, has been selected to validate the procedure here proposed. This event has been selected as it has resulted into a permanent fault for which the relevant faulted branch has been identified by the distribution network maintenance crew as the dotted "faulted line" reported in Fig. 7. The fault voltage transient, shown in Fig. 8, has been measured in correspondence of node 01 of Fig. 7.

The fault was interrupted by the overcurrent relay. Since the distribution network operates with an isolated neutral, the fault can be due to a three-phase or a phase-to-phase short circuit. The results shown in Fig. 8 can be used to distinguish between these two types of faults. Indeed, as this event produced large transients on only two phases, the occurrence of a phase-to-phase fault is inferred. Note that, for this specific fault type only the differential propagation mode is of interest [19], [22]. Therefore, the CWT analysis is applied to a transient obtained as the difference between the voltage signals of the two faulted phases (A and $B$ ).

Since the tower geometry of the overhead lines in this distribution network are the same as shown in [14], the differential propagation speed is determined as $2.941 \cdot 10^{8} \mathrm{~m} / \mathrm{s}$ for the identification of the characteristic frequencies defined by (1).

The characteristic frequency associated with the fault location is calculated as the interval of frequencies corresponding to the paths between the observation point, node 01 , and nodes 07 and 08 that delimit the faulted line (as shown by Fig. 7). Table III shows the characteristic frequencies calculated by (1).

Fig. 9 shows the obtained scalogram of the signal energy values $E_{\mathrm{cwt}}(a)$ provided by (3) by applying the CWT to the fault transient of Fig. 8. The scalogram of Fig. 9 is used to identify the characteristic frequencies also reported in Table III.

As shown by Table III, the identified fault location frequency is equal to $13.4 \mathrm{kHz}$ a value included in the interval between
TABLE III

CHARACTERISTIC AND CWT IDENTIFIED FREQUENCIES USING THE APPROACH of [11], [12] RELEVANT to THE PROPAGATION PATHS OF TOPOLOGIES REPORTED IN FIG. 7

\begin{tabular}{c|c|c|c}
\hline \hline Path & $\begin{array}{c}\text { Path length } \\
n_{p} \cdot L_{p} \\
(\mathrm{~km})\end{array}$ & $\begin{array}{c}\text { Theoretical } \\
\text { frequencies } f_{p, i} \\
\text { (traveling speed } \\
\text { equal to } \\
\left.2.94 \cdot 10^{8} \mathrm{~m} / \mathrm{s}\right) \\
(\mathrm{kHz})\end{array}$ & $\begin{array}{c}\text { CWT identified } \\
\text { frequencies } f_{p, i} \\
\text { by using the } \\
E_{c w t}(a) \\
(\mathrm{kHz})\end{array}$ \\
\hline Node 01-02 & $2 \times 2.79\left(n_{p}=2\right)$ & 52.65 & 50.25 \\
\hline Node 01-03 & $4 \times 1.76\left(n_{p}=4\right)$ & 41.88 & 38.35 \\
\hline Node 01-04 & $2 \times 2.34\left(n_{p}=2\right)$ & 62.81 & 62.10 \\
\hline Node 01-05 & $4 \times 2.90\left(n_{p}=4\right)$ & 25.35 & 25.95 \\
\hline Node 01-06 & $2 \times 9.03\left(n_{p}=2\right)$ & 16.28 & 18.25 \\
\hline Node 01-07 & $4 \times 4.79\left(n_{p}=4\right)$ & 15.35 & 13.40 \\
\hline Node 01-08 & $4 \times 6.43\left(n_{p}=4\right)$ & 11.44 & \\
\hline \hline
\end{tabular}

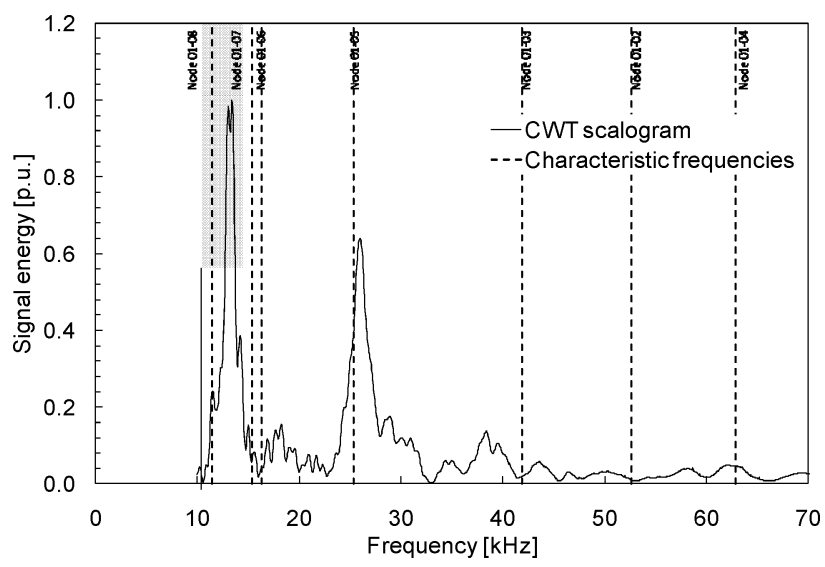

Fig. 9. Energy scalogram relevant to the CWT analysis of a fault transient related to a fault event occurred between node 07 and node 08 of the distribution network of Fig. 7.

the characteristic frequencies associated to the paths between nodes 01-07 and nodes 01-08, namely 11.44 and $15.35 \mathrm{kHz}$. By applying (1), such an identified frequency corresponds to a distance of $5.485 \mathrm{~km}$ from the measurement point node 01 .

Similar to the other cases, Fig. 10 shows the analysis relevant to the identification of time differences between local maxima of the coefficients $C(a, b)$ in the frequency range centered in the CWT identified frequencies $f_{p, i}^{l}=13.4 \mathrm{kHz}$ by using the $E_{\text {cwt }}(a)$. The results of Fig. 10, allows to determine a time difference between local maximum of $C(a, b)$ equal to $75.7 \mu$ s that corresponds to an improved identified characteristic frequency of $13.21 \mathrm{kHz}$. Such a frequency is also in the interval between the characteristic frequencies associated to nodes 07 and 08 , namely 11.44 and $15.35 \mathrm{kHz}$.

As a final comment, it is worth reminding that fault location procedures are conceived as post-fault processes performed after the relays intervention. The relevant computational time, however, should be limited anyway to avoid the delay of postfault restoration and reconfiguration operations. In this respect, a significant property of the proposed procedure is the independence of the computational time effort with respect to the network extension. As can be inferred from Section III, the computational time depends only to the following CWT parameters: 1) the duration of the analyzed transient and 2) the number of frequency intervals (or CWT scales $a$ ) adopted to discretize the 


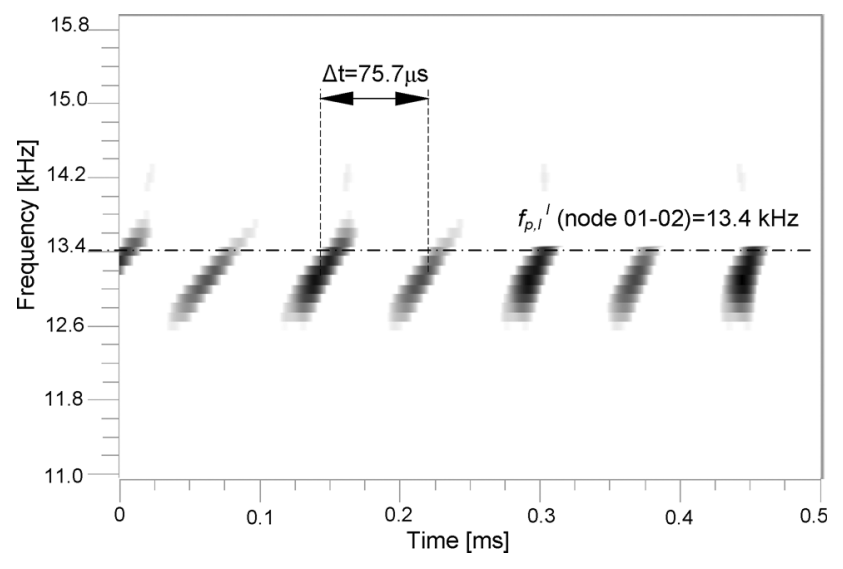

Fig. 10. Coefficients $C(a, b)$ obtained by means of the CWT analysis applied to the fault transient of Fig. 8: improved estimation of the characteristic frequency associated to the faulted path between nodes $01-07$ and $01-08$.

frequency spectrum. The results presented in this section have been obtained by adopting the following values:

$-N \cdot T_{s}$ equal to $1.5 \mathrm{~ms}$ (sampling frequency $1 / T_{s}=10$ $\mathrm{MHz}, N=15000)$;

- CWT frequency interval $2 \mathrm{kHz}-70 \mathrm{kHz}$ discretized in 136 decompositions $(\Delta f=500 \mathrm{~Hz})$.

The computational time required by the procedure on a 2.6 $\mathrm{GHz}$ Intel core 2 Duo PC is about $100 \mathrm{~s}$.

\section{CONCLUSION}

This paper has described a fault location procedure based on the CWT analysis of fault-originated transients. Compared to a procedure previously developed by the Authors, the one presented in this paper integrates both time and frequency information obtained from the CWT decompositions of fault transient signals. The proposed procedure consists of two steps: 1) initial estimation of the characteristic frequencies associated to the fault location by using the approach proposed in [14], [16] and 2) improvement of the estimate by identifying the time differences between local maximum of the signal coefficients $C(a, b)$ defined by the CWT (2) in the frequency range centered in correspondence of the previously identified frequencies.

The procedure is successfully applied to fault transient waveforms which are obtained experimentally during various fault scenarios, specially staged in reduced scale setup of a cable distribution network feeder. Test results confirm the improvement of the proposed procedure compared to the one presented in [14], [16] in terms of overall accuracy of the fault location.

Finally, the application of the proposed procedure to the transient recorded during a fault in a real distribution network with known characteristics has provided a convincing validation as well as an encouragement to pursuit in the further development of a fully automatic fault-location system to be installed in MV substations.

\section{ACKNOWLEDGMENT}

The paper takes into account the comments received during the presentation of a preliminary version at the 8 th International
Conference on Power System Transients (IPST'09), Kyoto, Japan June, 2-6, 2009.

\section{REFERENCES}

[1] Fault Management in Electrical Distribution Systems 1998, CIRED WG03 Fault management.

[2] IEEE Guide for Determining Fault Location on AC Transmission and Distribution Lines, IEEE Std C37.114, 2004

[3] A. G. Phadke and J. S. Thorp, Computer Relaying for Power Systems, 2nd ed. New York: Wiley, 2009.

[4] M. S. Sachdev and R. Agarwal, "A technique for estimating transmission line fault locations from digital impedance relay measurements," IEEE Trans. Power Del., vol. 3, no. 1, pp. 121-129, Jan. 1988

[5] K. Srinivasan and A. St.-Jacques, "A new fault location algorithm for radial transmission lines with loads," IEEE Trans. Power Del., vol. 4, no. 3, pp. 1676-1682, Jul. 1989.

[6] A. A. Girgis, D. G. Hart, and W. L. Peterson, "A new fault location technique for two- and three-terminal lines," IEEE Trans. Power Del., vol. 7, no. 1, pp. 98-107, Jan. 1992.

[7] M. El-Hami, L. L. Lai, D. J. Daruvala, and A. T. Johns, “A new travelling-wave based scheme for fault detection on overhead power distribution feeders," IEEE Trans. Power Del., vol. 7, no. 4, pp. 1825-1833, Oct. 1992.

[8] G. B. Ancell and N. C. Pahalawatha, "Maximum likelihood estimation of fault location on transmission lines using travelling waves," IEEE Trans. Power Del., vol. 9, no. 2, pp. 680-689, Apr. 1994.

[9] O. Chaari, M. Meunier, and F. Brouaye, "Wavelets: A new tool for resonant grounded power distribution systems relaying," IEEE Trans. Power Del., vol. 11, no. 3, pp. 1301-1308, Jul. 1996.

[10] F. H. Magnago and A. Abur, "Fault location using wavelets," IEEE Trans. Power Del., vol. 13, no. 4, pp. 1475-1480, Oct. 1998.

[11] F. H. Magnago and A. Abur, "A new fault location technique for radial distribution systems based on high frequency signals," in Proc. IEEE-Power Eng. Soc. Summer Meeting, Jul. 18-22, 1999, vol. 1, pp. 426-431.

[12] H. Hizman, P. A. Crossley, P. F. Gale, and G. Bryson, "Fault section identification and location on a distribution feeder using travelling waves," in 2002 IEEE Power Engineering Society Summer Meeting, Jul. 25-25, 2002, vol. 3, pp. 1107-1112.

[13] F. Yan, Z. Chen, Z. Liang, Y. Kong, and P. Li, "Fault location using wavelet packets," in Proc. Int. Conf. Power System Technology (PowerCon 2002), Oct. 13-17, 2002, vol. 4, pp. 2575-2579.

[14] A. Borghetti, S. Corsi, C. A. Nucci, M. Paolone, L. Peretto, and R. Tinarelli, "On the use of continuous-wavelet transform for fault location in distribution power networks," Elect. Power Energy Syst., vol. 28, pp. 608-617, 2006.

[15] F. Yan, T.-L. Ge, and H.-S. Zhao, "A new method of single-phase-toearth fault location for rural MV power networks," in Proc. Joint Int. Conf. Power System Technology and IEEE Power India Conf. (POWERCON 2008), Oct. 12-15, 2008.

[16] A. Borghetti, M. Bosetti, M. Di Silvestro, C. A. Nucci, and M. Paolone, "Continuous-wavelet transform for fault location in distribution power networks: Definition of mother wavelets inferred from fault originated transients," IEEE Trans. Power Syst., vol. 23, no. 2, pp. 380-388, May 2008.

[17] A. Graps, "An introduction to wavelets," IEEE Comput. Sci. Eng., vol. 2, no. 2, pp. 50-61, Summer 1995.

[18] T. Lobos, T. Sikorski, and P. Schegner, "Joint time-frequency representation of non-stationary signals in electrical power engineering," in Proc. 15th Power Systems Computation Conf. (PSCC'05), Liege, Belgium, Aug. 2005, paper fp 97

[19] H. W. Dommel, "Digital computer solution of electromagnetic transients in single and multi-phase networks," IEEE Trans. Power App. Syst., vol. 88, pp. 388-99, Apr. 1969.

[20] M. Kezunovic, M. Aganagic, V. Skendzic, J. Domaszewicz, J. K Bladow, D. M. Hamai, and S. M. McKenna, "Transients computation for relay testing in real-time," IEEE Trans. Power Del., vol. 9, no. 3, pp. 1298-1307, Jul. 1994.

[21] H. Y. Li, Z. Q. Bo, B. Caunce, and S. Potts, "A fault transient comparison technique for multi-ended distribution feeders," in Proc. 7th Int. Conf. Developments in Power System Protection (IEE), Apr. 9-12, 2001, pp. 153-156.

[22] E. Clarke, Circuit Analysis of AC Power Systems. New York: Wiley, 1943, vol. 1.

[23] Assessment of Emission Limits for the Connection of Distorting Installations to MV, HV and EHV Power Systems 2008, IEC/TR 61000-3-6. 
[24] K. Yamabuki, A. Borghetti, F. Napolitano, C. A. Nucci, M. Paolone, L. Peretto, R. Tinarelli, M. Bernardi, and R. Vitale, "A distributed measurement system for correlating faults to lightning in distribution networks," in Proc. Int. Symp. High Voltage Engineering, Ljubljana, Slovenia, Aug. 27-31, 2007.

[25] A. Borghetti, F. Napolitano, C. A. Nucci, M. Paolone, M. Bernardi, F. Rachidi, and K. Yamabuki, "Correlation of lightning events and faults in distribution power networks: A joint research project," in Proc. Cigre Session 2008, Paris, France, Aug. 24-29, 2008, paper C4-117.

[26] M. Bernardi, C. Giorgi, and V. Biscaglia, "Medium voltage line faults correlation with lightning events recorded with the Italian LLP system CESI-SIRF," in Proc. 24th Int. Conf. Lightning Protection, Birmingham, U.K., 1998, vol. 1, pp. 187-192.

Alberto Borghetti (M'97-SM'03) was born in Cesena, Italy, in 1967. He graduated (Hons.) in electrical engineering from the University of Bologna, Bologna, Italy, in 1992.

Since then, he has been with the Power System Group, University of Bologna, where he was appointed a Researcher in 1994 and Associate Professor of Electric Power Systems in 2004. His main research interests concern power system analysis, with particular reference to voltage collapse, power system restoration after blackout, electromagnetic transients, and optimal generation scheduling.

Mauro Bosetti was born in Trento, Italy, in 1978. He received the M.S. (Hons.) and $\mathrm{Ph} . \mathrm{D}$. degrees in electrical engineering from the University of Bologna, Bologna, Italy, in 2005 and 2009, respectively.

His research interests are distributed generation, with particular reference to micro-grid operation, and fault location.

Carlo Alberto Nucci (M'91-SM'02-F'07) was born in Bologna, Italy, in 1956. $\mathrm{He}$ received the electrical engineering degree (Hons.) from the University of Bologna in 1982.
Currently, he is a Professor of Electrical Power Systems in the Department of Electrical Engineering, University of Bologna. He is author or coauthor of more than 200 scientific papers published in reviewed journals or presented at international conferences. His research interests include power systems transients and dynamics, with particular reference to lightning impact on power lines, system restoration after blackout, and distributed generation.

Prof. Nucci is a member of the IEEE Working Group "Lightning performance of Distribution lines". He is Chairman of the CIGRE Study Committee C4 "System Technical Performance" and a Fellow of the Institution of Engineering and Technology (IET). Since 2010, he has the Editor-in-Chief of the Electric Power System Research journal. He is now serving as Region 8 Representative of the IEEE Power Engineering Society (PES).

Mario Paolone (M'07) was born in Campobasso, Italy, in 1973. He received the electrical engineering (Hons.) and Ph.D. degrees from the University of Bologna, Bologna, Italy, in 1998 and 2002, respectively.

Currently, he is a Researcher within the Power Systems Group, University of Bologna. His research interests are power system transients, with particular reference to LEMP interaction with electrical networks, power systems dynamics, power system protection, and smart grids. He is author or co-author of over 100 scientific publications in reviewed journals and international conferences.

Dr. Paolone is a member of the IEEE Working Group "Lightning Performance of Distribution Lines" and of the joint CIGRE-CIRED Working Group "Protection of MV and LV Networks Against Lightning" and Co-Chairperson of the Technical Program Committee of the 8th edition of the International Conference on Power Systems Transients, Kyoto, Japan, 2009.

Ali Abur (F'03) received the B.S. degree from Orta Dou Teknik Universitesi, Ankara, Turkey, in 1979 and the M.S. and Ph.D. degrees from the Ohio State University, Columbus, in 1981 and 1985, respectively.

He was a Professor from late 1985 until November 2005 with the Department of Electrical Engineering, Texas A\&M University, College Station. Since then, he has been with the Department of Electrical and Computer Engineering, Northeastern University, Boston, MA. 